\title{
Sand Dune Species Distribution and Size Variations in Two Areas Inside a Natural Protected Area Subjected to Different Human Disturbance
}

\author{
Loretta Gratani, Maria Fiore Crescente, Laura Varone, Emanuela Gini, Carlo Ricotta, Andrea Bonito
}

Department of Environmental Biology, Sapienza University of Rome, Rome, Italy.

Email: loretta.gratani@uniroma1.it

Received June $28^{\text {th }}, 2013$; revised July $30^{\text {th }}, 2013$; accepted August $15^{\text {th }}, 2013$

Copyright (C) 2013 Loretta Gratani et al. This is an open access article distributed under the Creative Commons Attribution License, which permits unrestricted use, distribution, and reproduction in any medium, provided the original work is properly cited.

\begin{abstract}
Sand dune species were analyzed across two areas included in the "Roman Coastal State Nature Reserve" (Italy): Ostia (O) and Marina di Palidoro (P). Significant variations in species distribution, dry mass and size over the gradient from the water-edge toward the inland between $\mathrm{O}$ and $\mathrm{P}$ were observed. Species colonize $\mathrm{O}$, on an average, at $79 \mathrm{~m}$ from the water-edge extended along the gradient toward the inland over a length of $26 \mathrm{~m}$. PD is $40.8 \pm 9.9$ plants $\cdot \mathrm{m}^{-2}$. Ononis variegata and Elymus farctus have the highest PD $\left(15.4 \pm 1.3\right.$ plants $\left.\cdot \mathrm{m}^{-2}\right)$, followed by Silene colorata and Sporobulus pungens $\left(4.3 \pm 3.9\right.$ plants $\left.\cdot \mathrm{m}^{-2}\right)$, by Cakile maritima, Echinophora spinosa, Eryngium maritimum, Ammophila arenaria, Anthemis maritima, Cyperus capitatus, Medicago marina and Calystegia soldanella $\left(<0.5\right.$ plants $\left.\mathrm{m}^{-2}\right)$, with $C$. soldanella having the lowest $\mathrm{PD}\left(0.02\right.$ plants $\left.\mathrm{m}^{-2}\right)$. On an average, in $\mathrm{P}$, the species colonize the dune at $17 \mathrm{~m}$ from the water-edge for a length of $46 \mathrm{~m}$ toward the inland, $\mathrm{PD}$ is on an average $21.5 \pm 16.1 \mathrm{plants}^{\prime} \mathrm{m}^{-2}$. S. pungens, E. farctus and O. variegata have the highest PD $\left(6.0 \pm 2.4\right.$ plants $\left.\mathrm{m}^{-2}\right)$, followed by C. capitatus $\left(2.1 \pm 4.7\right.$ plants $\left.\mathrm{m}^{-2}\right)$, by $S$. colorata and A. maritima $\left(0.4 \pm 0.1\right.$ plants $\left.\mathrm{m}^{-2}\right)$ and by E. spinosa, E. maritimum, M. marina, Pancratium maritimum and C. soldanella $\left(0.2\right.$ plants $\left.\cdot \mathrm{m}^{-2}\right)$. The results underline a larger species presence along the gradient from the water-edge toward the inland in $\mathrm{P}$ site compared to $\mathrm{O}$ site where, on the contrary, plants exclusively colonize the inner dune area due to the strong human disturbance which causes the foredune to become flat. Nevertheless, the presence of the most important autoctonous sand dune species (on an average, $15.3 \pm 0.5$ species) can provide information for restoring the perturbed dune areas when preparing management strategies considering that the maintenance of coastal areas depends on the maintenance of native species.
\end{abstract}

Keywords: Dune Vegetation; Human Disturbance; Plant Density; Organic Soil Matter

\section{Introduction}

Some critical factors affect the survival and distribution of coastal sand dune species which grow on a physiologically dried substrate characterised by a low mineral content [1-4]. A factor which contributes to select dune species is the climatic effect on sand movements $[5,6]$ such as wind-speed entrainment thresholds for sand particles and salt burial $[7,8]$. With regard to species assemblage, dune-builder plants may grow on fore-dunes, burial-tolerant plants on inter-dunes and shrubs on stabilized dunes [9]. Morphological and physiological plant adaptations are important especially on fore-dunes [10-12] where few species are capable of withstanding the stress factors imposed by limited environmental resources and recurrent disturbance. On older dunes, where salt spray, nutrient and water are no longer exclusive limiting factors, competition for space and light may affect species richness [13-15]. Plant species presence is also related to organic matter which varies from water-edge toward the inland $[11,13]$.

The ecological state of sea coasts is often critical worldwide [16]. In Europe most of the well conserved coastal dune areas are at present under protection [17] and are included in the EU Directive Habitat 92/43/CEE. Nevertheless, dunes are the most threatened habitats by the expansion of urban areas and the development of seaside tourism $[9,18]$ leading to the fragmentation of vegetation and the disappearance of vegetation bands developing on mobile dunes [19]. Disturbance is defined as a stochastic event in opposition to environmental stress which is predictable and rather continuous [20]. 
Most common environmental stress factors are drought and soil nutrient deficiency [21]. Among human disturbance, infrastructure development is widespread on coasts in Europe. Furthermore, landscape fragmentation disrupts large scale geo-morphological processes [22] and mechanical damages through trampling reduce or even destroy vegetation [13]. Increased knowledge of plant species response to stress factors (i.e. climate, soil, anthropogenic interference) aims at the long-term land use planning to ensure sustainability of coastal resources while providing management flexibility for the future [23].

The main objective of this research was to compare the species presence in two areas included in a natural protected area developing along the Tyrrhenian coast near Rome and subjected to a different human disturbance. Considering the importance of biological diversity maintenance [24-27], we analyzed variations in sand dune species presence, dry mass and size over the gradient from the water-edge toward the inland.

\section{Methods}

\subsection{The Study Area}

The study was carried out in the year 2012 in the Roman Coastal State Nature Reserve (Italian decree of 1996) which extended for ca $43 \mathrm{Km}$ along the Tyrrhenian coast near Rome (Italy). Two areas were selected: Ostia $\left(41^{\circ} 41^{\prime} 00^{\prime \prime} \mathrm{N} 12^{\circ} 22^{\prime} 39^{\prime \prime} \mathrm{E}\right)$ and Marina di Palidoro $\left(41^{\circ} 54^{\prime} 43^{\prime \prime N} 12^{\circ} 08^{\prime} 47^{\prime \prime E}\right.$ ) (Figure 1). Despite the two areas being under protection, in recent years they were subjected to human disturbance. In particular, Ostia (O) was a small populated city $\left(85,301\right.$ people $/ \mathrm{km}^{2}$, data from Rome Municipality for the year 2010) where numerous buildings and bathing establishments were built in the last 50 years. Two sub-areas were selected for measurements: $\mathrm{O}_{1}\left(41^{\circ} 40^{\prime} 58^{\prime \prime} \mathrm{N}, 12^{\circ} 22^{\prime} 40^{\prime \prime} \mathrm{E}\right)$ near a gully, extending along the coast line for ca $200 \mathrm{~m}$, and $\mathrm{O}_{2}$ $\left(41^{\circ} 40^{\prime} 37^{\prime \prime} \mathrm{N}, 12^{\circ} 23^{\prime} 14 " \mathrm{E}\right)$ near a bathing establishment, extending along the coastline for ca $400 \mathrm{~m}$. At both $\mathrm{O}_{1}$ and $\mathrm{O}_{2}$ the sand dune appeared flat up to $70-80 \mathrm{~m}$ from the water-edge, followed by a mobile dune area characterised by a moderate slope of ca $6 \%$, which finished with the fixed dune colonized by Mediterranean shrubs. The distance from the water-edge to the shrubby layer was $109 \mathrm{~m}$ and $100 \mathrm{~m}$ in $\mathrm{O}_{1}$ and $\mathrm{O}_{2}$, respectively. Dune species were present at 85 and $73 \mathrm{~m}$ from the water-edge in $\mathrm{O}_{1}$ and $\mathrm{O}_{2}$, respectively.

Marina di Palidoro (P) was characterized by a lower human disturbance than $\mathrm{O}$ because of few buildings and bathing establishments. Two sub-areas were selected in $\mathrm{P}$ for measurements: $\mathrm{P}_{1}\left(41^{\circ} 55^{\prime} 06^{\prime \prime} \mathrm{N}, 12^{\circ} 08^{\prime} 17^{\prime \prime} \mathrm{E}\right)$ near the hospital Bambino Gesù, extending along the coast line for ca $1600 \mathrm{~m}$, and $\mathrm{P}_{2}\left(41^{\circ} 54^{\prime} 43^{\prime \prime} \mathrm{N}, 12^{\circ} 08^{\prime} 46 " \mathrm{E}\right)$ near a block of flats, extending along the coast line for ca $700 \mathrm{~m}$. In $\mathrm{P}_{1}$ and $\mathrm{P}_{2}$ the drift line and the foredune extended along the gradient from the water-edge toward the inland for ca $33 \mathrm{~m}$ and $41 \mathrm{~m}$, respectively, was characterized by a moderate slope (ca $2 \%$ ). Then it was followed by a mobile dune area extending for ca $20 \mathrm{~m}$ and $32 \mathrm{~m}$ in $\mathrm{P}_{1}$ and $\mathrm{P}_{2}$, respectively, and by the fixed dune with Mediterranean shrubs. The distance from the water-edge to the

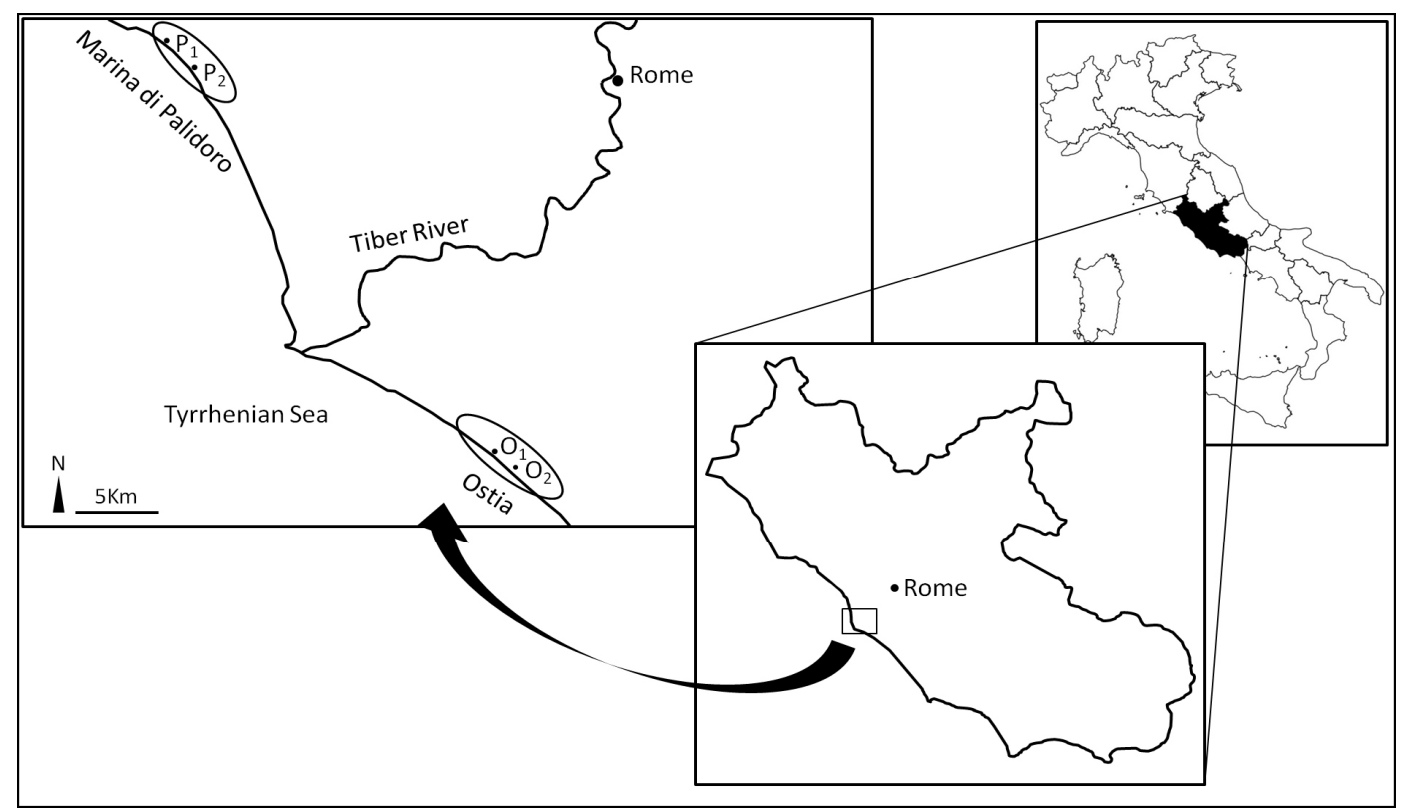

Figure 1. The two studied areas along the Thyrrenian coast near Rome, Ostia $\left(O, 41^{\circ} 41^{\prime} 00^{\prime \prime} \mathrm{N}, 1^{\circ} 22^{\prime} 39^{\prime \prime} \mathrm{E}\right)$ and Marina di Palidoro ( $\left.\mathrm{P}, 4^{\circ} \mathbf{5 4}^{\prime} 43^{\prime \prime} \mathrm{N}, \mathbf{1 2}^{\circ} 08^{\prime} 47^{\prime \prime} \mathrm{E}\right)$, and the considered sub-areas $\left(\mathrm{O}_{1}, \mathrm{O}_{2}, \mathrm{P}_{1}\right.$ and $\left.\mathrm{P}_{2}\right)$ are indicated. 
shrubby layer was $53 \mathrm{~m}$ and $73 \mathrm{~m}$ for $\mathrm{P}_{1}$ and $\mathrm{P}_{2}$, respectively. Species were present at 20 and $15 \mathrm{~m}$ from the water-edge, in $\mathrm{P}_{1}$ and $\mathrm{P}_{2}$ respectively.

A transect oriented from the water-edge toward the inland was established in each of the selected sub-areas $\left(\mathrm{O}_{1}, \mathrm{O}_{2}, \mathrm{P}_{1}, \mathrm{P}_{2}\right)$ for vegetation sampling. Along each transect, plots $(10 \times 10 \mathrm{~m}$ each) were established, $3 \mathrm{~m}$ apart, starting from the water-edge to the inland (i.e. Mediterranean shrubby). Plot numbers were established in relation to the extension in length of the dune from water edge toward the inland and to species presence. In each sub-area the first plot was established where the first species presence occurred. In particular, two plots were established in $\mathrm{O}_{1}\left(\mathrm{O}_{1 \mathrm{a}}\right.$, and $\mathrm{O}_{1 \mathrm{~b}}$ at 85 and $98 \mathrm{~m}$ from the water edge, respectively), $\mathrm{O}_{2}\left(\mathrm{O}_{2 \mathrm{a}}, \mathrm{O}_{2 \mathrm{~b}}\right.$ at $73 \mathrm{~m}$ and 86 $\mathrm{m}$ from the water edge, respectively), and in $\mathrm{P}_{1}\left(\mathrm{P}_{1 \mathrm{a}}\right.$ and $\mathrm{P}_{1 \mathrm{~b}}$ at 20 and $33 \mathrm{~m}$ from the water-edge, respectively), and four plots in $\mathrm{P}_{2}\left(\mathrm{P}_{2 \mathrm{a}}, \mathrm{P}_{2 \mathrm{~b}}, \mathrm{P}_{2 \mathrm{c}}\right.$ and $\mathrm{P}_{2 \mathrm{~d}}$ at 15, 28, 41 and $54 \mathrm{~m}$ from the water-edge, respectively).

\subsection{Climate and Microclimate}

The selected areas were characterised by a Mediterranean type of climate. At $\mathrm{O}$, the total annual rainfall was 589 $\mathrm{mm}$, the mean minimum air temperature of the coldest months (January and February) was $4.8^{\circ} \mathrm{C}$ and the mean maximum air temperature of the warmest months (July and August) was $29.1^{\circ} \mathrm{C}$ (data from the Meteorological Station of Pratica di Mare, for the years 2000-2012) (Figure 2). At $\mathrm{P}$, the total annual rainfall was $556 \mathrm{~mm}$, the mean minimum air temperature of the coldest months (January and February) was $3.2^{\circ} \mathrm{C}$ and the mean maximum air temperature of the warmest months (July and August) was $29.6^{\circ} \mathrm{C}$ (data from the Meteorological Station of Fiumicino, for the years 2000-2012) (Figure 2). The dominant winds were from $\mathrm{W}$ and the others from $\mathrm{S}$ and SE. The mean yearly winds speed was 16.6 knots and 18.7 knots at $\mathrm{O}$ and $\mathrm{P}$, respectively [28].

Microclimate was measured in the considered sub-areas at 20,40,60,80, $100 \mathrm{~m}$ from the water-edge toward the inland, periodically (twice a month) during the study period. In each sampling occasion, air temperature ( $\mathrm{T}$, ${ }^{\circ} \mathrm{C}$ ), relative air humidity $(\mathrm{RH}, \%)$, total irradiance (I, $\mu$ mol photon $\mathrm{m}^{-2} \cdot \mathrm{s}^{-1}$ ) and wind speed $(\mathrm{W}, \mathrm{m} / \mathrm{s})$ were recorded at $50 \mathrm{~cm}$ from the sand level. On each sampling occasion, measurements were carried out at 12:00 a.m.
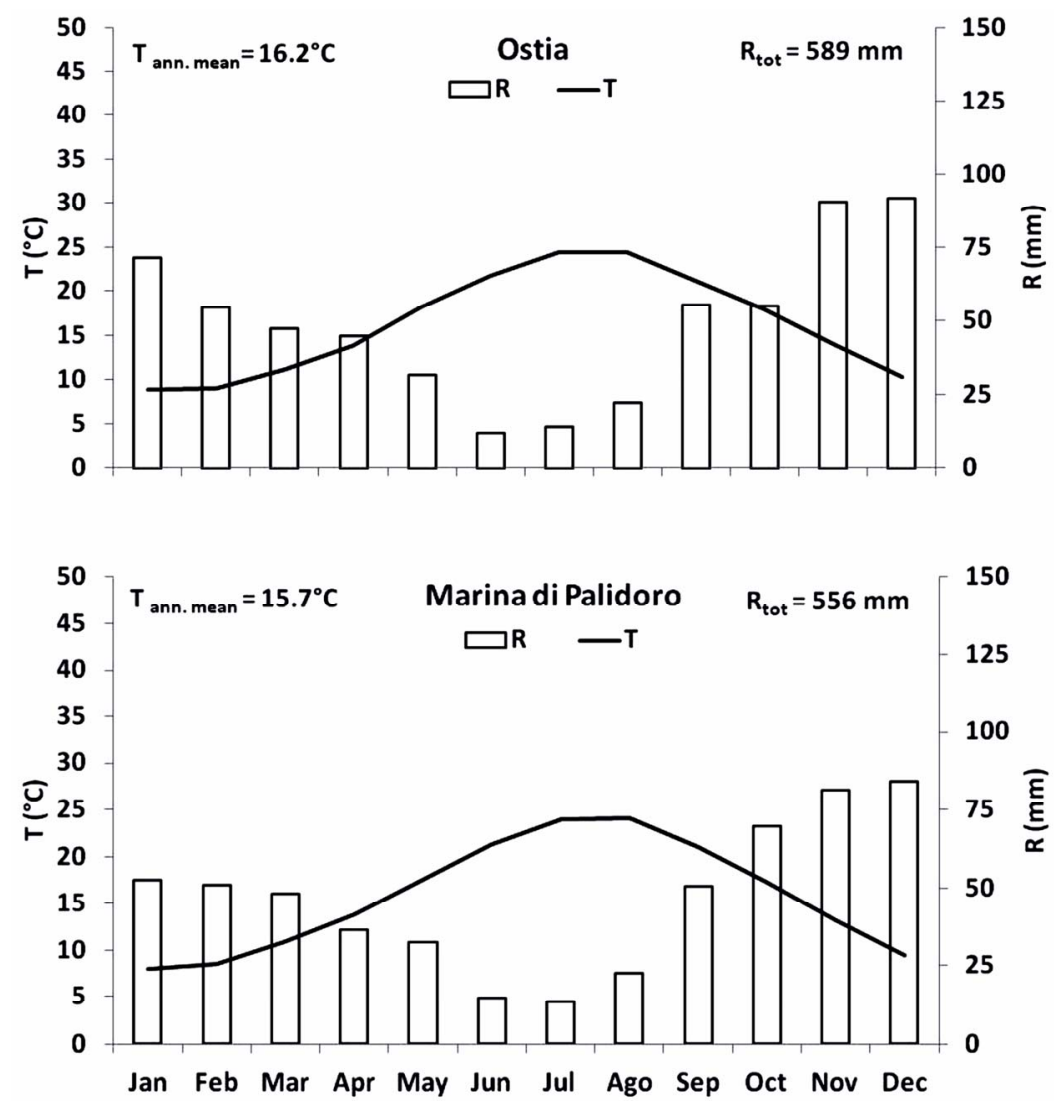

Figure 2. Climate diagrams of Ostia (O) and Marina di Palidoro (P) (data from the Meteorological Station of Pratica di Mare and of Fiumicino, respectively, for the period 2000-2011). Total monthly rainfall ( $R$, mm, columns), mean monthly air temperature $\left(T,{ }^{\circ} \mathrm{C}\right.$, lines), annual mean temperature $\left(T_{\text {ann.mean }}\right)$ and total annual rainfall $\left(R_{\text {tot }}\right)$ are shown. 
Air temperature and air humidity were measured by a thermo-hygrometer (HD8901, Delta Ohm, I), total irradiance by a radiometer (LI-185B with a 190SB Quantum Sensor, LI-COR, USA) and wind speed by an anemometer (LUTRON AM-4201).

\subsection{Sand Characterization}

Triplicate sand samples were collected in the considered sub-areas at 20, 40,60, 80, $100 \mathrm{~m}$ from the water-edge, for determination of sand water content (SWC, \%), $\mathrm{pH}$, soil organic matter (SOM, \%) content and total nitrogen (N, \%) content. All sand samples were collected at the end of May (at least 5 days after the last rainfall) at a depth of $40 \mathrm{~cm}$ by a drill. Sand samples were transported immediately to the laboratory. Sand samples were air dried at room temperature and then passed through $2 \mathrm{~mm}$ sieve. SWC was determined on sand samples (500 g each) as fresh sand minus dry sand divided by dry sand percent, calculated after oven-dried at $90^{\circ} \mathrm{C}$ to a constant mass. The $\mathrm{pH}$ was measured with a glass electrode in a suspension of sand in deionized water. SOM was determined colorimetrically with potassium dichromate, according to [29] and the $\mathrm{N}$ content by Kjeldahl method, according to [30].

\subsection{Plant Species Presence and Plant Traits}

Species presence was recorded in the considered plots from May to June, corresponding to the maximum plant biomass [31,32]. The number of plants per species was counted in each plot to calculate plant density (PD, individuals $\cdot \mathrm{m}^{-2}$ ).

Measurements of plant traits were carried out on representative plant species ( 5 plants per species in each plot) at the beginning of June. It included plant height $(\mathrm{H}, \mathrm{m})$, total plant volume per plot $\left(\mathrm{V}, \mathrm{cm}^{3} \cdot \mathrm{m}^{-2}\right)$, total plant area per unit of covered area at sand level $\left(\mathrm{PA}, \mathrm{cm}^{2} \cdot \mathrm{m}^{-2}\right)$ and total aboveground plant biomass (TPB, $\mathrm{g} \cdot \mathrm{m}^{-2}$ ). Plant material was harvested, oven dried and then weighed to obtain dry mass (DM, g), according to [33]. TPB per species was calculated by multiplying DM and PD. H was defined as the maximum vertical distance from the sand level to the highest point of the plant. V was calculated by the volume of a cylinder, according to [34].

\subsection{Statistical Analysis}

The main gradients in species composition were extracted by ordination (principal coordinate analysis, PcoA) which was performed on the plot-to-plot dissimilarity matrix and calculated with the Jaccard coefficient for species presence and absence data.

Differences of the means for the considered traits were tested by one-way ANOVA, and Tukey test for multiple comparisons. Kolmogorov-Smirnov and Levene tests were used to verify the assumption of normality and homogeneity of variances, respectively.

All statistical tests were performed by using Statistica 6.0 (Statsoft, USA). All data were shown as mean \pm S.D.

\section{Results}

\subsection{Microclimate and Sand Characterization}

The microclimate of the considered sub-areas $\left(\mathrm{P}_{1}\right.$ and $\mathrm{P}_{2}$ and $\mathrm{O}_{1}$ and $\mathrm{O}_{2}$ ) is shown in Figure 3. In all the considered sub-areas, $\mathrm{T}$ increased by $15 \%$ from water-edge to the inland, while RH and I decreased by $13 \%, 6 \%$ respectively (mean of $\mathrm{P}_{1}, \mathrm{P}_{2}, \mathrm{O}_{1}$ and $\mathrm{O}_{2}$ ). The wind (W) action decreased, on an average, $56 \%$ from water-edge toward the inland, both in $\mathrm{O}$ and $\mathrm{P}$ sub-areas.

The $\mathrm{O}$ and $\mathrm{P}$ sand characterization is shown in Figure 4. In the considered sub-areas, SWC and $\mathrm{pH}$ decreased on an average, $18 \%$ and $11 \%$ from the water-edge toward the inland, respectively, while SOM and $\mathrm{N}$ content increased more than $100 \%$ and $113 \%$, respectively (mean of $\mathrm{O}$ and $\mathrm{P})$.

\subsection{Plant Traits}

Data on species presence, PD, H, V, PA and TPB in the considered plots are shown in Table 1. The number of species was larger in $\mathrm{P}\left(15.5 \pm 0.5\right.$, mean of $\mathrm{P}_{1}$ and $\mathrm{P}_{2}$ plots) than in $\mathrm{O}\left(14.5 \pm 0.5\right.$, mean of $\mathrm{O}_{1}$ and $\mathrm{O}_{2}$ plots $)$. A larger $\mathrm{PD}$ was monitored in $\mathrm{P}_{1 \mathrm{~b}}$, and $\mathrm{P}_{2 \mathrm{c}},(37.7 \pm 3.1$ plants $\cdot \mathrm{m}^{-2}$, mean value $)$ and in $\mathrm{O}_{1 \mathrm{~b}}$ and $\mathrm{O}_{2 \mathrm{~b}}(48.8 \pm 0.15$ plants $\cdot \mathrm{m}^{-2}$, mean value) than in the other plots $(22.1 \pm$ 14.3 plants $\cdot \mathrm{m}^{-2}$, mean value). Crucianella maritima (PD $=0.53$ plants $\cdot \mathrm{m}^{-2}$, mean value) was monitored only in $\mathrm{P}_{2 \mathrm{~d}}$. The plots farer from the water-edge had a higher $\mathrm{H}$ than those closer to it. In particular, $\mathrm{H}$ was on an average $59 \%$ higher in $\mathrm{O}_{1 b}$ than in $\mathrm{O}_{1 \mathrm{a}}$ and $70 \%$ higher in $\mathrm{O}_{2 \mathrm{~b}}$ than in $\mathrm{O}_{2 \mathrm{a}}$. $\mathrm{H}$ was $41 \%$ higher in $\mathrm{P}_{1 \mathrm{~b}}$ than in $\mathrm{P}_{1 \mathrm{a}}$, and $74 \%$ higher in $\mathrm{P}_{2 \mathrm{c}}$ and $\mathrm{P}_{2 \mathrm{~d}}$ than in $\mathrm{P}_{2 \mathrm{a}}$ and $\mathrm{P}_{2 \mathrm{~b}}$. On an average, PA was $82 \%$ larger in $\mathrm{O}_{1 \mathrm{~b}}$ than in $\mathrm{O}_{1 \mathrm{a}}$ and $94 \%$ larger in $\mathrm{O}_{2 \mathrm{~b}}$ than in $\mathrm{O}_{2 \mathrm{a}}$. $\mathrm{PA}$ was $266 \%$ larger in $\mathrm{P}_{1 \mathrm{~b}}$ than in $\mathrm{P}_{1 \mathrm{a}}$, and $577 \%$ larger in $\mathrm{P}_{2 \mathrm{c}}$ and $\mathrm{P}_{2 \mathrm{~d}}$ than in $\mathrm{P}_{2 \mathrm{a}}$ and $\mathrm{P}_{2 \mathrm{~b}}$. TPB ranged from $1145.8 \mathrm{~g} \cdot \mathrm{m}^{-2}$ (in $\mathrm{O}$ ) to $1413.0 \mathrm{~g} \cdot \mathrm{m}^{-2}$ (in $\mathrm{P}$ ) and it was the highest in $\mathrm{O}_{2 \mathrm{~b}}\left(304.9 \mathrm{~g} \cdot \mathrm{m}^{-2}\right)$ among $\mathrm{O}$ plots, and in $\mathrm{P}_{2 \mathrm{~d}}$ $\left(394.4 \mathrm{~g} \cdot \mathrm{m}^{-2}\right)$ among P plots.

As regards to the species, E. farctus had the highest TPB and V $\left(141.6 \pm 62.2 \mathrm{~g} \cdot \mathrm{m}^{-2}\right.$ and $19837 \pm 8885$ $\mathrm{cm}^{3} \cdot \mathrm{m}^{-2}$ respectively, mean of the considered $\mathrm{P}$ and $\mathrm{O}$ plots) and Chamaesyce peplis the lowest one (0.008 \pm $0.010 \mathrm{~g} \cdot \mathrm{m}^{-2}$ and $1.9 \pm 2.1 \mathrm{~cm}^{3} \cdot \mathrm{m}^{-2}$ respectively, mean of the considered $\mathrm{P}$ and $\mathrm{O}$ plots).

\subsection{Principal Coordinate Analysis}

PCoA extracted two factors accounting for $53.33 \%$ of the 

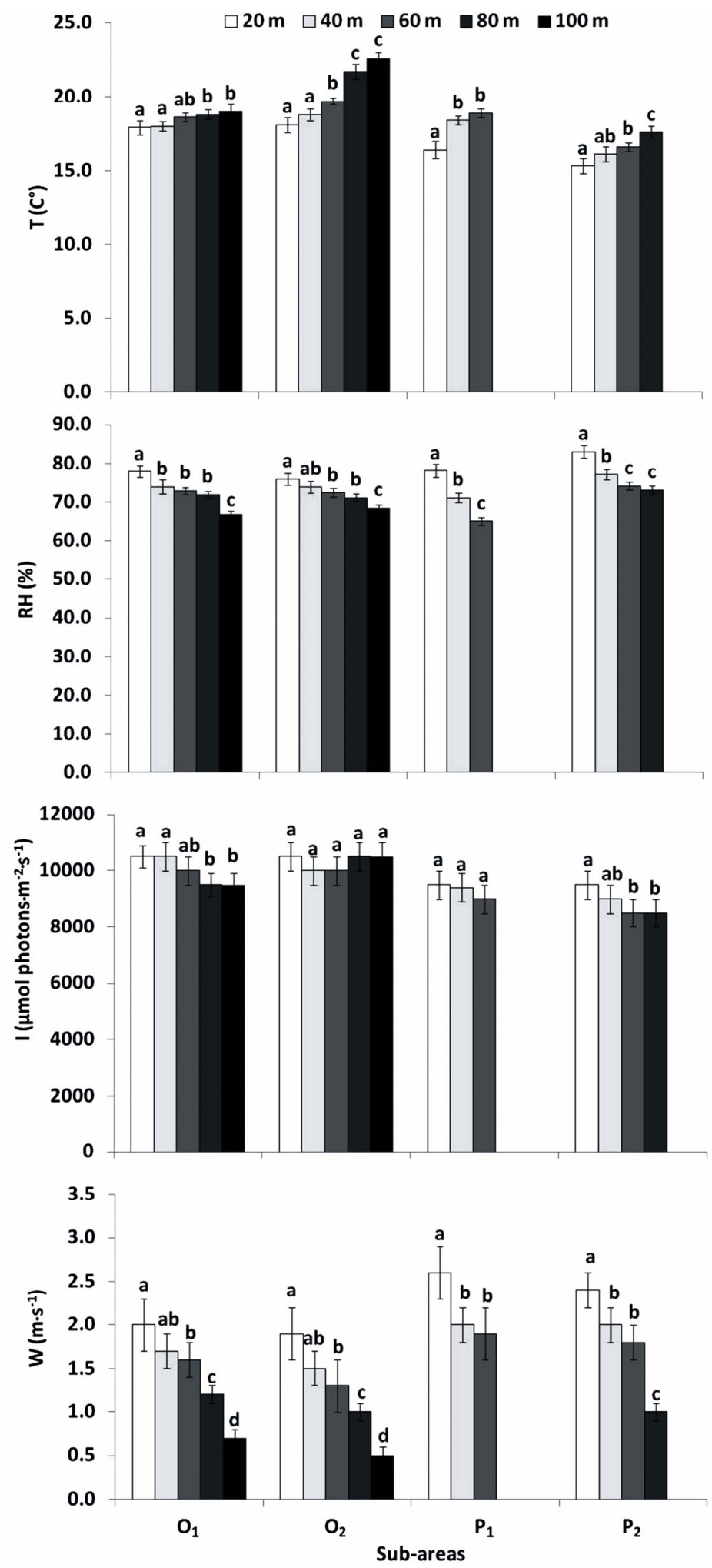

Figure 3. Air temperature $\left(\mathrm{T},{ }^{\circ} \mathrm{C}\right)$, relative air humidity $(\mathrm{RH}, \%)$, total irradiance $\left(\mathrm{I}, \mu \mathrm{mol}\right.$ photons $\left.\mathrm{m}^{-2} \cdot \mathrm{s}^{-1}\right)$ and wind $\mathrm{speed}$

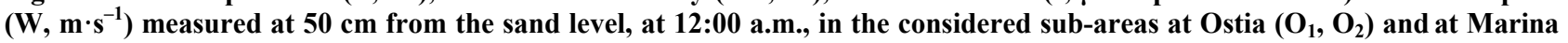
di Palidoro $\left(P_{1}\right.$ and $\left.P_{2}\right)$. Measurements were carried out at 20, 40, 60, 80, $100 \mathrm{~m}$ from water-edge. Mean values \pm standard deviation are shown. For each sub-area different letters indicate significant differences among the distances over the gradient from the water-edge toward the inland. 

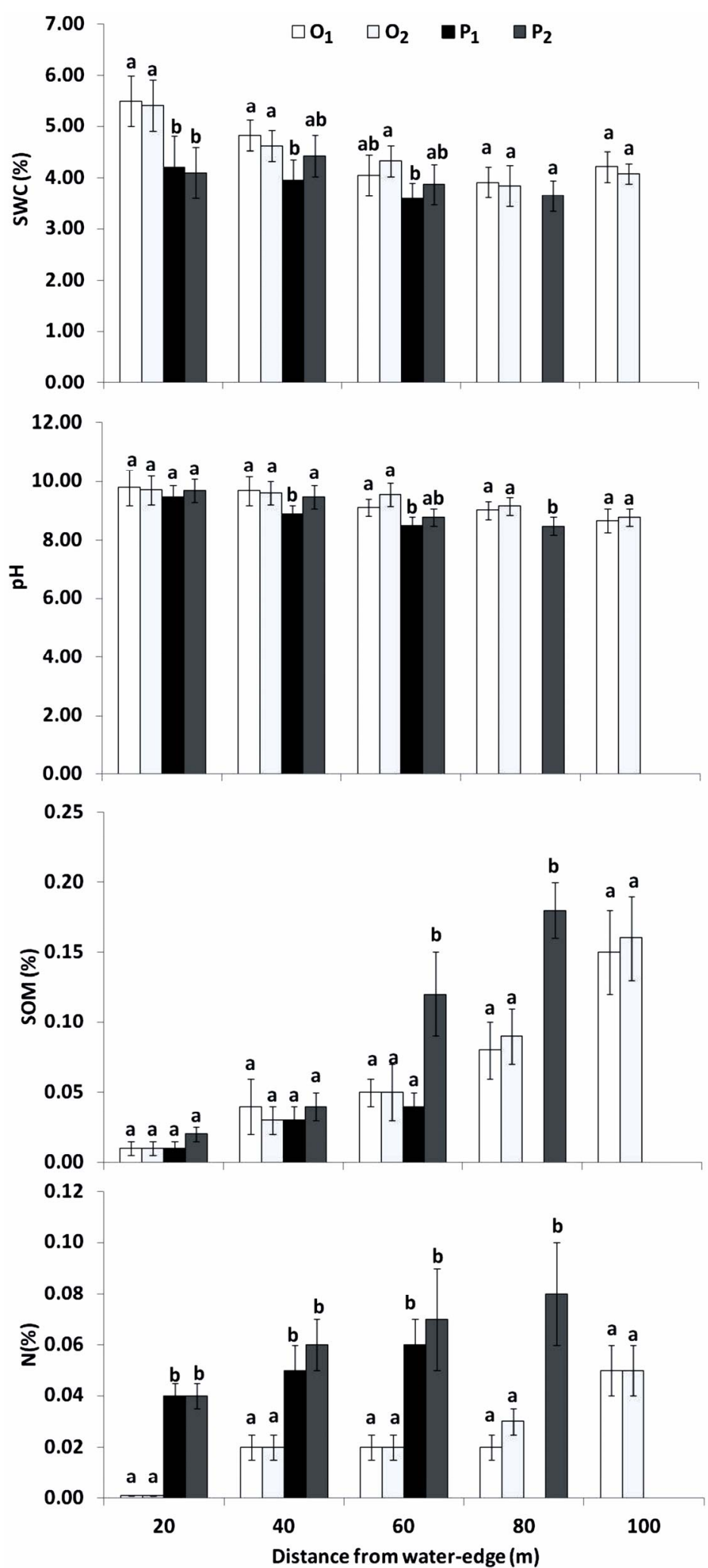

Figure 4. Sand water content (SWC, \%), pH, soil organic matter (SOM, \%) and total nitrogen content (N, \%) collected in the considered sub-areas in Ostia $\left(\mathrm{O}_{1}, \mathrm{O}_{2}\right)$ and Marina di Palidoro $\left(\mathrm{P}_{1}\right.$ and $\left.\mathrm{P}_{2}\right)$ at 20, 40, 60, 80, $100 \mathrm{~m}$ from water-edge. Mean values \pm standard deviation are shown. For each distance over the gradient from the water-edge toward the inland sub-area different letters indicate significant differences among the sub-areas. 
Table 1. Data on species presence, plant density, plant height, plant area, plant volume and total plant biomass of the considered plots used in this study. For each species different letters indicate significant differences among the plots.

\begin{tabular}{|c|c|c|c|c|c|c|c|c|c|c|}
\hline Distance (m) & $85-95$ & $98-108$ & $73-83$ & $86-96$ & $20-30$ & $33-43$ & $15-25$ & $28-38$ & $41-51$ & $54-64$ \\
\hline $\begin{array}{ll}\text { Species } & \text { Plot } \\
\end{array}$ & $\mathbf{O}_{1 \mathrm{a}}$ & $\mathbf{O}_{1 \mathrm{~b}}$ & $\mathbf{O}_{2 \mathrm{a}}$ & $\mathbf{O}_{2 \mathrm{~b}}$ & $\mathbf{P}_{1 \mathrm{a}}$ & $\mathbf{P}_{1 \mathrm{~b}}$ & $\mathbf{P}_{2 \mathrm{a}}$ & $\mathbf{P}_{2 b}$ & $\mathbf{P}_{2 c}$ & $\mathbf{P}_{2 \mathrm{~d}}$ \\
\hline & \multicolumn{2}{|c|}{$\mathrm{PD}\left(\right.$ plant $\left.\cdot \mathrm{m}^{-2}\right)$} & \multicolumn{2}{|c|}{$\mathrm{PD}\left(\right.$ plant $\left.\cdot \mathrm{m}^{-2}\right)$} & \multicolumn{2}{|c|}{$\mathrm{PD}\left(\right.$ plant $\left.\cdot \mathrm{m}^{-2}\right)$} & \multicolumn{4}{|c|}{$\mathrm{PD}\left(\right.$ plant $\left.\cdot \mathrm{m}^{-2}\right)$} \\
\hline Cakile maritima Scop. & $0.01 \mathrm{a}$ & - & - & $0.01 \mathrm{a}$ & $0.02 \mathrm{a}$ & - & $0.88 \mathrm{~b}$ & - & $0.02 \mathrm{a}$ & - \\
\hline Echinophora spinosa $\mathrm{L}$. & $0.05 \mathrm{a}$ & $0.12 \mathrm{~b}$ & $0.07 \mathrm{a}$ & $0.11 \mathrm{~b}$ & $0.02 \mathrm{a}$ & $0.07 \mathrm{a}$ & - & $0.02 \mathrm{a}$ & $0.16 \mathrm{c}$ & $0.25 \mathrm{~d}$ \\
\hline Eryngium maritimum $\mathbf{L}$. & - & $0.01 \mathrm{a}$ & - & $0.10 \mathrm{~b}$ & $0.07 \mathrm{ab}$ & $0.03 \mathrm{a}$ & - & $0.15 \mathrm{c}$ & - & $0.15 \mathrm{c}$ \\
\hline $\begin{array}{l}\text { Elymus farctus (Viv.) Runemark } \\
\text { ex Melderis }\end{array}$ & $18.60 \mathrm{a}$ & $12.09 \mathrm{~b}$ & $20.70 \mathrm{a}$ & $14.00 \mathrm{~b}$ & $11.85 \mathrm{~b}$ & $5.15 \mathrm{c}$ & - & $9.00 \mathrm{~d}$ & $13.15 \mathrm{~b}$ & $5.04 \mathrm{c}$ \\
\hline Ammophila arenaria (L.) Link & - & $1.16 \mathrm{a}$ & - & $0.80 \mathrm{ac}$ & - & $0.14 \mathrm{~b}$ & - & - & $0.54 \mathrm{c}$ & - \\
\hline Anthemis maritimaL. & $0.26 \mathrm{a}$ & $0.39 \mathrm{~b}$ & $0.14 \mathrm{a}$ & $0.58 \mathrm{~b}$ & $0.05 \mathrm{c}$ & $1.36 \mathrm{~d}$ & - & - & $0.18 \mathrm{a}$ & $0.59 \mathrm{~b}$ \\
\hline Cyperus capitatus Vand. & - & $0.59 \mathrm{ab}$ & - & $0.31 \mathrm{a}$ & - & $0.79 \mathrm{~b}$ & - & - & - & $11.75 \mathrm{c}$ \\
\hline Ononis variegata $\mathrm{L}$. & $0.14 \mathrm{a}$ & $28.3 \mathrm{~b}$ & - & $29.40 \mathrm{~b}$ & - & $13.25 \mathrm{c}$ & - & - & - & $6.02 \mathrm{~d}$ \\
\hline Medicago marinaL. & - & $0.15 \mathrm{a}$ & $0.02 \mathrm{~b}$ & $0.02 \mathrm{~b}$ & - & $0.04 \mathrm{~b}$ & - & - & - & $0.12 \mathrm{a}$ \\
\hline Crucianella maritimaL. & - & - & - & - & - & - & - & - & - & 0.53 \\
\hline Calystegia soldanella(L.) R. Br. & $0.02 \mathrm{a}$ & - & - & - & - & $0.03 \mathrm{a}$ & - & - & - & $0.06 \mathrm{~b}$ \\
\hline Sporobolus pungens(Schreb.) Kunth & $16.55 \mathrm{a}$ & $2.85 \mathrm{~b}$ & $7.85 \mathrm{c}$ & $1.20 \mathrm{~d}$ & - & $13.00 \mathrm{e}$ & - & $0.15 \mathrm{f}$ & $25.75 \mathrm{~g}$ & $5.50 \mathrm{c}$ \\
\hline Silene colorataPoir. & $0.71 \mathrm{a}$ & $3.10 \mathrm{~b}$ & $0.04 \mathrm{c}$ & $2.45 \mathrm{~b}$ & - & $0.92 \mathrm{~d}$ & - & - & - & $1.43 \mathrm{e}$ \\
\hline Pancratium maritimumL. & - & $0.01 \mathrm{a}$ & - & $0.01 \mathrm{a}$ & $0.03 \mathrm{ac}$ & $0.71 \mathrm{~b}$ & - & - & $0.01 \mathrm{a}$ & $0.05 \mathrm{c}$ \\
\hline Salsola kaliL. & $0.12 \mathrm{a}$ & - & $0.01 \mathrm{~b}$ & - & $0.02 \mathrm{~b}$ & - & $0.07 \mathrm{c}$ & $0.12 \mathrm{a}$ & $0.04 \mathrm{bc}$ & - \\
\hline Chamaesyce peplis (L.) Prokh. & $0.01 \mathrm{a}$ & - & $0.01 \mathrm{a}$ & - & $0.01 \mathrm{a}$ & - & - & - & $0.08 \mathrm{~b}$ & - \\
\hline \multirow[t]{2}{*}{ Total } & 17.54 & 48.76 & 28.82 & 48.96 & 12.04 & 35.46 & 0.07 & 9.43 & 39.91 & 31.46 \\
\hline & \multicolumn{2}{|c|}{$\mathrm{H}(\mathrm{cm})$} & \multicolumn{2}{|c|}{$\mathrm{H}(\mathrm{cm})$} & \multicolumn{2}{|c|}{$\mathrm{H}(\mathrm{cm})$} & \multicolumn{4}{|c|}{$\mathrm{H}(\mathrm{cm})$} \\
\hline Cakile maritima Scop. & $22.0 \mathrm{a}$ & - & - & $35.0 \mathrm{~b}$ & $11.5 \mathrm{c}$ & - & $16.5 \mathrm{~d}$ & - & $19.3 \mathrm{~d}$ & - \\
\hline Echinophora spinosa $\mathrm{L}$. & $40.6 \mathrm{a}$ & $36.5 \mathrm{a}$ & $29.5 \mathrm{~b}$ & $29.1 \mathrm{~b}$ & $10.8 \mathrm{c}$ & $21.0 \mathrm{~d}$ & - & $28.5 \mathrm{~b}$ & $26.6 \mathrm{~b}$ & $17.2 \mathrm{e}$ \\
\hline Eryngium maritimum L. & - & $22.5 \mathrm{a}$ & - & $26.4 \mathrm{~b}$ & $21.8 \mathrm{ac}$ & $19.3 \mathrm{c}$ & - & $12.3 \mathrm{~d}$ & - & $24.9 \mathrm{ab}$ \\
\hline $\begin{array}{l}\text { Elymus farctus (Viv.) Runemark } \\
\text { ex Melderis }\end{array}$ & $43.0 \mathrm{a}$ & $43.0 \mathrm{a}$ & $40.5 \mathrm{a}$ & $64.8 \mathrm{~b}$ & $43.7 \mathrm{a}$ & $43.5 \mathrm{a}$ & - & $53.7 \mathrm{c}$ & $44.0 \mathrm{a}$ & $41.0 \mathrm{a}$ \\
\hline Ammophila arenaria (L.) Link & - & $92.5 \mathrm{a}$ & - & $105.0 \mathrm{~b}$ & - & $28.0 \mathrm{c}$ & - & - & $108.1 \mathrm{~b}$ & - \\
\hline Anthemis maritimaL. & $16.3 \mathrm{a}$ & $16.9 \mathrm{ab}$ & $19.5 \mathrm{~b}$ & $14.6 \mathrm{a}$ & $13.1 \mathrm{a}$ & $17.4 \mathrm{ab}$ & - & - & $20.2 \mathrm{~b}$ & $13.3 \mathrm{a}$ \\
\hline Cyperus capitatus Vand. & - & $39.0 \mathrm{a}$ & - & $37.8 \mathrm{a}$ & - & $36.0 \mathrm{a}$ & - & - & - & $44.5 \mathrm{~b}$ \\
\hline Ononis variegataL. & $10.1 \mathrm{a}$ & $11.5 \mathrm{a}$ & - & $9.5 \mathrm{a}$ & - & $9.5 \mathrm{a}$ & - & - & - & $11.5 \mathrm{a}$ \\
\hline Medicago marinaL. & - & $12.4 \mathrm{a}$ & $7.8 \mathrm{~b}$ & $9.3 \mathrm{~b}$ & - & $12.6 \mathrm{a}$ & - & - & - & $15.6 \mathrm{a}$ \\
\hline Crucianella maritimaL. & - & - & - & - & - & - & - & - & - & 18.2 \\
\hline Calystegia soldanella(L.) R. Br. & $3.5 \mathrm{a}$ & - & - & - & - & $3.5 \mathrm{a}$ & - & - & - & $6.5 \mathrm{~b}$ \\
\hline Sporobolus pungens(Schreb.) Kunth & $19.0 \mathrm{ac}$ & $19.0 \mathrm{ac}$ & $17.0 \mathrm{a}$ & $16.0 \mathrm{a}$ & - & $10.5 \mathrm{~b}$ & - & $10.1 \mathrm{~b}$ & $11.0 \mathrm{~b}$ & $21.5 \mathrm{c}$ \\
\hline Silene colorataPoir. & $14.0 \mathrm{a}$ & $15.5 \mathrm{a}$ & $20.0 \mathrm{~b}$ & $14.5 \mathrm{a}$ & - & $14.0 \mathrm{a}$ & - & - & - & $20.0 \mathrm{~b}$ \\
\hline Pancratium maritimumL. & - & $15.5 \mathrm{a}$ & - & $17.0 \mathrm{a}$ & $10.6 \mathrm{~b}$ & $28.5 \mathrm{c}$ & - & - & $38.0 \mathrm{~d}$ & $41.7 \mathrm{~d}$ \\
\hline Salsola kaliL. & $10.4 \mathrm{a}$ & - & $9.8 \mathrm{a}$ & - & $2.3 \mathrm{~b}$ & - & $2.0 \mathrm{~b}$ & $2.3 \mathrm{~b}$ & $2.4 \mathrm{~b}$ & - \\
\hline Chamaesyce peplis (L.) Prokh. & $4.0 \mathrm{a}$ & - & $4.5 \mathrm{a}$ & - & $2.0 \mathrm{~b}$ & - & - & - & $2.4 \mathrm{~b}$ & - \\
\hline \multirow[t]{2}{*}{ Mean } & 18.3 & 29.5 & 18.6 & 31.6 & 14.5 & 20.3 & 9.3 & 21.4 & 30.2 & 23.0 \\
\hline & \multicolumn{2}{|c|}{$\mathrm{PA}\left(\mathrm{cm}^{2} \cdot \mathrm{m}^{-2}\right)$} & \multicolumn{2}{|c|}{$\mathrm{PA}\left(\mathrm{cm}^{2} \cdot \mathrm{m}^{-2}\right)$} & \multicolumn{2}{|c|}{$\mathrm{PA}\left(\mathrm{cm}^{2} \cdot \mathrm{m}^{-2}\right)$} & \multicolumn{4}{|c|}{$\mathrm{PA}\left(\mathrm{cm}^{2} \cdot \mathrm{m}^{-2}\right)$} \\
\hline Cakile maritima Scop. & $15.00 \mathrm{a}$ & - & - & $13.35 \mathrm{a}$ & $4.00 \mathrm{~b}$ & - & $352.00 \mathrm{c}$ & - & $81.63 \mathrm{~d}$ & - \\
\hline Echinophora spinosa $\mathbf{L}$. & $136.45 \mathrm{a}$ & $321.71 \mathrm{~b}$ & $99.63 \mathrm{c}$ & $158.77 \mathrm{~d}$ & $3.14 \mathrm{e}$ & $22.02 \mathrm{f}$ & - & $4.50 \mathrm{e}$ & $232.55 \mathrm{~g}$ & $109.86 \mathrm{c}$ \\
\hline Eryngium maritimum $\mathbf{L}$. & - & $4.02 \mathrm{a}$ & - & $231.43 \mathrm{~b}$ & $22.79 \mathrm{c}$ & $10.59 \mathrm{c}$ & - & $49.28 \mathrm{~d}$ & - & $104.26 \mathrm{e}$ \\
\hline $\begin{array}{l}\text { Elymus farctus (Viv.) Runemark } \\
\text { ex Melderis }\end{array}$ & $672.00 \mathrm{a}$ & $423.15 \mathrm{~b}$ & $724.50 \mathrm{a}$ & $490.00 \mathrm{~b}$ & $414.75 \mathrm{~b}$ & $180.25 \mathrm{c}$ & - & $315.00 \mathrm{~d}$ & $460.25 \mathrm{~b}$ & $176.40 \mathrm{c}$ \\
\hline Ammophila arenaria (L.) Link & - & $29.05 \mathrm{a}$ & - & $20.00 \mathrm{a}$ & - & $3.38 \mathrm{~b}$ & - & - & $181.03 \mathrm{c}$ & - \\
\hline Anthemis maritimaL. & $413.90 \mathrm{a}$ & $524.19 \mathrm{~b}$ & $358.68 \mathrm{a}$ & $654.05 \mathrm{c}$ & $187.08 \mathrm{~d}$ & $1415.94 \mathrm{e}$ & - & - & $626.01 \mathrm{c}$ & $887.86 \mathrm{f}$ \\
\hline Cyperus capitatus Vand. & - & $28.08 \mathrm{ab}$ & - & $14.64 \mathrm{a}$ & - & $37.68 \mathrm{~b}$ & - & - & - & $564.00 \mathrm{c}$ \\
\hline
\end{tabular}


Continued

\begin{tabular}{|c|c|c|c|c|c|c|c|c|c|c|}
\hline Ononis variegata $\mathrm{L}$. & $41.99 \mathrm{a}$ & $707.50 \mathrm{~b}$ & - & $735.00 \mathrm{~b}$ & - & $331.25 \mathrm{c}$ & - & - & - & $138.00 \mathrm{~d}$ \\
\hline Medicago marinaL. & - & $507.74 \mathrm{a}$ & $12.56 \mathrm{~b}$ & $97.50 \mathrm{c}$ & - & $104.8 \mathrm{c}$ & - & - & - & $495.73 \mathrm{a}$ \\
\hline Crucianella maritimaL. & - & - & - & - & - & - & - & - & - & 498.45 \\
\hline Calystegia soldanella(L.) R. Br. & $0.29 \mathrm{a}$ & - & - & - & - & $1.05 \mathrm{a}$ & - & - & - & $11.00 \mathrm{~b}$ \\
\hline Sporobolus pungens(Schreb.) Kunth & $136.80 \mathrm{a}$ & $22.80 \mathrm{~b}$ & $62.80 \mathrm{c}$ & $9.60 \mathrm{~d}$ & - & $104.00 \mathrm{e}$ & - & $1.20 \mathrm{f}$ & $206.00 \mathrm{~g}$ & $44.00 \mathrm{c}$ \\
\hline Silene colorataPoir. & $4.26 \mathrm{a}$ & $18.60 \mathrm{~b}$ & $0.21 \mathrm{c}$ & $14.70 \mathrm{~b}$ & - & $5.49 \mathrm{a}$ & - & - & - & $8.55 \mathrm{~d}$ \\
\hline Pancratium maritimumL. & - & $1.63 \mathrm{a}$ & - & $2.72 \mathrm{a}$ & $1.47 \mathrm{a}$ & $101.52 \mathrm{~b}$ & - & - & $14.23 \mathrm{c}$ & $65.89 \mathrm{~d}$ \\
\hline Salsola kaliL. & $0.82 \mathrm{a}$ & - & $0.08 \mathrm{~b}$ & - & $0.10 \mathrm{~b}$ & - & $1.48 \mathrm{c}$ & $0.96 \mathrm{a}$ & $0.38 \mathrm{~d}$ & - \\
\hline Chamaesyce peplis (L.) Prokh. & $0.16 \mathrm{a}$ & - & $0.29 \mathrm{a}$ & - & $0.28 \mathrm{a}$ & - & - & - & $2.12 \mathrm{~b}$ & - \\
\hline \multirow[t]{2}{*}{ Total } & 1421.66 & 2588.46 & 1258.76 & 2441.75 & 633.61 & 2317.96 & 353.48 & 370.94 & 1804.20 & 3104.01 \\
\hline & \multicolumn{2}{|c|}{$\mathrm{V}\left(\mathrm{cm}^{3} \cdot \mathrm{m}^{-2}\right)$} & \multicolumn{2}{|c|}{$\mathrm{V}\left(\mathrm{cm}^{3} \cdot \mathrm{m}^{-2}\right)$} & \multicolumn{2}{|c|}{$\mathrm{V}\left(\mathrm{cm}^{3} \cdot \mathrm{m}^{-2}\right)$} & \multicolumn{4}{|c|}{$\mathrm{V}\left(\mathrm{cm}^{3} \cdot \mathrm{m}^{-2}\right)$} \\
\hline Cakile maritima Scop. & $330.0 \mathrm{a}$ & - & - & $467.1 \mathrm{~b}$ & $46.0 \mathrm{c}$ & - & $5808.0 \mathrm{~d}$ & - & $1571.3 \mathrm{e}$ & - \\
\hline Echinophora spinosa $\mathrm{L}$. & $5543.3 \mathrm{a}$ & $11742.2 \mathrm{~b}$ & $2941.2 \mathrm{c}$ & $4626.5 \mathrm{~d}$ & $33.8 \mathrm{e}$ & $461.5 \mathrm{f}$ & - & $128.3 \mathrm{~g}$ & $6194.0 \mathrm{~h}$ & $1892.8 \mathrm{i}$ \\
\hline Eryngium maritimum L. & - & $90.5 \mathrm{a}$ & - & $6114.4 \mathrm{~b}$ & $495.8 \mathrm{c}$ & $204.8 \mathrm{~d}$ & - & 606.9 e & - & $2598.5 \mathrm{f}$ \\
\hline $\begin{array}{l}\text { Elymus farctus (Viv.) Runemark } \\
\text { ex Melderis }\end{array}$ & $28896.0 \mathrm{a}$ & $18195.5 \mathrm{~b}$ & $29342.3 \mathrm{a}$ & $31752.0 \mathrm{a}$ & $18106.8 \mathrm{~b}$ & $7840.9 \mathrm{c}$ & - & $16915.5 \mathrm{~b}$ & $20251.0 \mathrm{~b}$ & $7232.4 \mathrm{c}$ \\
\hline Ammophila arenaria (L.) Link & - & $2686.7 \mathrm{a}$ & - & $2100.0 \mathrm{a}$ & - & $94.5 \mathrm{~b}$ & - & - & $19576.7 \mathrm{c}$ & - \\
\hline Anthemis maritimaL. & $6736.7 \mathrm{a}$ & $8862.5 \mathrm{~b}$ & 7008.6 a & $9569.7 \mathrm{~b}$ & $2447.6 \mathrm{c}$ & $24605.4 \mathrm{~d}$ & - & - & $12629.8 \mathrm{e}$ & $11801.1 \mathrm{e}$ \\
\hline Cyperus capitatus Vand. & - & $1095.1 \mathrm{ab}$ & - & $552.7 \mathrm{~b}$ & - & $1356.5 \mathrm{a}$ & - & - & - & $25098.0 \mathrm{c}$ \\
\hline Ononis variegata $\mathrm{L}$. & $425.6 \mathrm{a}$ & $8136.3 \mathrm{~b}$ & - & $6982.5 \mathrm{~b}$ & - & $3146.9 \mathrm{c}$ & - & - & - & $1587.0 \mathrm{~d}$ \\
\hline Medicago marinaL. & - & $6315.7 \mathrm{a}$ & $97.3 \mathrm{~b}$ & $901.9 \mathrm{c}$ & - & $1320.4 \mathrm{~d}$ & - & - & - & 7736.9 a \\
\hline Crucianella maritimaL. & - & - & - & - & - & - & - & - & - & 9090.7 \\
\hline Calystegia soldanella(L.) R. Br. & $1.0 \mathrm{a}$ & - & - & - & - & $3.7 \mathrm{a}$ & - & - & - & $71.5 \mathrm{~b}$ \\
\hline Sporobolus pungens(Schreb.) Kunth & $2599.2 \mathrm{a}$ & $433.2 \mathrm{~b}$ & $1067.6 \mathrm{c}$ & $153.6 \mathrm{~d}$ & - & $1092.0 \mathrm{c}$ & - & $12.1 \mathrm{e}$ & $2266.0 \mathrm{f}$ & $946.0 \mathrm{c}$ \\
\hline Silene colorataPoir. & $59.6 \mathrm{a}$ & $288.3 \mathrm{~b}$ & $4.2 \mathrm{c}$ & $213.2 \mathrm{~b}$ & - & 76.9 a & - & - & - & $171.0 \mathrm{~d}$ \\
\hline Pancratium maritimumL. & - & $25.2 \mathrm{a}$ & - & $46.2 \mathrm{a}$ & $15.6 \mathrm{a}$ & $2893.3 \mathrm{~b}$ & - & - & $540.7 \mathrm{c}$ & $2747.5 \mathrm{~b}$ \\
\hline Salsola kaliL. & $8.6 \mathrm{a}$ & - & $0.8 \mathrm{~b}$ & - & $0.2 \mathrm{~b}$ & - & $3.0 \mathrm{c}$ & $2.3 \mathrm{c}$ & $0.9 \mathrm{~b}$ & - \\
\hline Chamaesyce peplis (L.) Prokh. & $0.6 \mathrm{a}$ & - & $1.3 \mathrm{a}$ & - & $0.6 \mathrm{a}$ & - & - & - & $5.1 \mathrm{~b}$ & - \\
\hline \multirow[t]{2}{*}{ Total } & 44600.6 & $\mathbf{5 7 8 7 1 . 1}$ & 40463.2 & 63479.7 & 21146.4 & 43096.7 & 5811.0 & 17665.0 & 63035.3 & 70973.5 \\
\hline & \multicolumn{2}{|c|}{ TPB $\left(\mathrm{g} \cdot \mathrm{m}^{-2}\right)$} & \multicolumn{2}{|c|}{ TPB $\left(\mathrm{g} \cdot \mathrm{m}^{-2}\right)$} & \multicolumn{2}{|c|}{$\mathrm{TPB}\left(\mathrm{g} \cdot \mathrm{m}^{-2}\right)$} & \multicolumn{4}{|c|}{$\mathrm{TPB}\left(\mathrm{g} \cdot \mathrm{m}^{-2}\right)$} \\
\hline Cakile maritima Scop. & $0.250 \mathrm{a}$ & - & - & $0.222 \mathrm{a}$ & $0.067 \mathrm{~b}$ & - & $5.870 \mathrm{c}$ & - & $1.361 \mathrm{~d}$ & - \\
\hline Echinophora spinosa $\mathrm{L}$. & $4.107 \mathrm{a}$ & $9.683 \mathrm{~b}$ & $2.999 \mathrm{c}$ & $4.779 \mathrm{a}$ & $0.120 \mathrm{e}$ & $0.662 \mathrm{f}$ & - & $0.120 \mathrm{e}$ & $7.000 \mathrm{~g}$ & $3.307 \mathrm{c}$ \\
\hline Eryngium maritimum L. & - & $0.257 \mathrm{a}$ & - & $14.808 \mathrm{~b}$ & $1.460 \mathrm{c}$ & $0.678 \mathrm{ac}$ & - & $3.155 \mathrm{~d}$ & - & $6.671 \mathrm{e}$ \\
\hline $\begin{array}{l}\text { Elymus farctus (Viv.) Runemark } \\
\text { ex Melderis }\end{array}$ & $216.318 \mathrm{a}$ & $140.607 \mathrm{~b}$ & $240.741 \mathrm{a}$ & $162.820 \mathrm{~b}$ & $137.816 \mathrm{~b}$ & $59.895 \mathrm{c}$ & - & $104.67 \mathrm{~d}$ & $152.935 \mathrm{~b}$ & $58.615 \mathrm{c}$ \\
\hline Ammophila arenaria (L.) Link & - & $20.308 \mathrm{a}$ & - & $13.984 \mathrm{a}$ & - & $2.360 \mathrm{c}$ & - & - & $126.574 \mathrm{~d}$ & - \\
\hline Anthemis maritimaL. & $27.417 \mathrm{a}$ & $34.724 \mathrm{~b}$ & $23.760 \mathrm{a}$ & $43.325 \mathrm{c}$ & $12.393 \mathrm{~d}$ & $93.795 \mathrm{e}$ & - & - & $41.468 \mathrm{c}$ & $58.814 \mathrm{f}$ \\
\hline Cyperus capitatus Vand. & - & $3.463 \mathrm{ab}$ & - & $1.806 \mathrm{a}$ & - & $4.647 \mathrm{~b}$ & - & - & - & $69.560 \mathrm{c}$ \\
\hline Ononis variegata $\mathrm{L}$. & $2.872 \mathrm{a}$ & $48.393 \mathrm{~b}$ & - & $50.274 \mathrm{~b}$ & - & $22.658 \mathrm{c}$ & - & & - & $10.294 \mathrm{~d}$ \\
\hline Medicago marinaL. & - & $34.770 \mathrm{a}$ & $0.860 \mathrm{~b}$ & $6.677 \mathrm{c}$ & - & $7.177 \mathrm{c}$ & - & - & - & $33.947 \mathrm{a}$ \\
\hline Crucianella maritimaL. & - & - & - & - & - & - & - & - & - & 41.335 \\
\hline Calystegia soldanella(L.) R. Br. & $0.007 \mathrm{a}$ & - & - & - & - & $0.025 \mathrm{a}$ & - & - & - & $0.262 \mathrm{~b}$ \\
\hline Sporobolus pungens(Schreb.) Kunth & $15.391 \mathrm{a}$ & $2.651 \mathrm{~b}$ & $7.301 \mathrm{c}$ & $1.116 \mathrm{~d}$ & - & $12.090 \mathrm{e}$ & - & $0.140 \mathrm{f}$ & $23.948 \mathrm{~g}$ & $5.115 \mathrm{bc}$ \\
\hline Silene colorataPoir. & $0.234 \mathrm{a}$ & $1.023 \mathrm{~b}$ & $0.012 \mathrm{c}$ & $0.809 \mathrm{bd}$ & - & $0.630 \mathrm{de}$ & - & - & - & 0.470 ae \\
\hline Pancratium maritimumL. & - & $2.614 \mathrm{a}$ & - & $4.375 \mathrm{~b}$ & $2.316 \mathrm{a}$ & $163.306 \mathrm{c}$ & - & - & $22.888 \mathrm{~d}$ & $105.987 \mathrm{e}$ \\
\hline Salsola kaliL. & $0.047 \mathrm{~cd}$ & - & $0.006 \mathrm{a}$ & - & $0.006 \mathrm{a}$ & - & $0.084 \mathrm{~b}$ & $0.063 \mathrm{bc}$ & $0.022 \mathrm{~d}$ & - \\
\hline Chamaesyce peplis (L.) Prokh. & $0.002 \mathrm{a}$ & - & $0.003 \mathrm{a}$ & - & $0.003 \mathrm{a}$ & - & - & - & $0.023 \mathrm{~b}$ & - \\
\hline Total & 266.646 & 298.493 & 275.680 & 304.995 & 154.180 & 367.922 & 5.954 & 108.150 & 376.218 & 394.378 \\
\hline
\end{tabular}


total variance. Factor 1 and 2 accounted for $34.80 \%$ and $18.53 \%$ of the total variance, respectively. The first factor reflected the turnover of the vegetation along the sea-inland vegetation zonation, with higher scores of the factor indicating species closer to the water-edge. Variation in species composition among plots was greater in the longer and less disturbed transect of $\mathrm{P}\left(\mathrm{P}_{2}\right)$ as underlined by a greater dispersion of the plots in this sub-area along the second ordination axis (Figure 5).

\section{Discussion}

The natural vegetation of the Tyrrhenian coast which includes "The Roman Coast State Nature Reserve" until the years 1970-1980 was characterised by different vegetation bands. In particular, after the aphytoic dune area, the first community on the strandline was characterized by Ca. maritima, E. peplis and Salsola kali, followed by a second community on the foredune with $E$. farctus, S. pungens, and C. capitatus. The third community which contributed to dune consolidation was characterized by $A$. arenaria, E. spinosa, O. variegata, E. maritimum and $M$. marina and the inner community by Cr. maritima and P. maritimum, followed by the Mediterranean maquis $[9,35]$.

In stressful environments, species are patchily distributed [36-38], however, spatial species aggregation may also be caused by within-community environmental heterogeneity and preference for common micro-habitats [39].
Our results on the whole show significant variations of species presence and density between Ostia and Marina di Palidoro in response to the different human disturbance. The strong human disturbance at Ostia is the result of the large presence of beach establishments, numerous buildings, the intense use of mechanical means to clean the area and a strong human trampling all year long, which have largely altered the sand dune, and in particular the foredune. In response to these factors, species colonize the dune area, on an average, at $79 \mathrm{~m}$ from the water-edge (mean of $\mathrm{O}_{1}$ and $\mathrm{O}_{2}$ ) extending along the gradient toward the inland over a length of $26 \mathrm{~m}$ (mean value). $\mathrm{PD}$ is $40.8 \pm 9.9$ plants $\cdot \mathrm{m}^{-2}$ (mean of all the considered $\mathrm{O}$ plots). In particular, O. variegata and E. farctus have the highest PD $\left(15.4 \pm 1.3\right.$ plants $\cdot \mathrm{m}^{-2}$ mean value), followed by $S$. colorata and $S$. pugens $(4.3 \pm 3.9$ plants $\cdot \mathrm{m}^{-2}$, mean value), by Ca. maritima, E. spinosa, E. maritimum, A. arenaria, A. maritima, C. capitatus, $M$. marina and $C$. soldanella (lower than 0.5 plants $\cdot \mathrm{m}^{-2}$ ). $C$. soldanella is a prostrate plant with long rhizomes that survives seawater inundation better than many other species [40] which causes the aerial parts to die but the buried rhizome may survive. This species co-occurs with $E$. farctus in many other foredune areas in Europe [41]. $\mathrm{H}$ and TPB vary from $18.3 \mathrm{~m}$ to $31.6 \mathrm{~m}$ and from 266.7 $\mathrm{g} \cdot \mathrm{m}^{-2}$ to $305.0 \mathrm{~g} \cdot \mathrm{m}^{-2}$, respectively.

The lower human disturbance at $\mathrm{P}$ in respect to $\mathrm{O}$, determines the species presence, on an average, at $14.5 \mathrm{~m}$ from the water-edge extending along the gradient toward

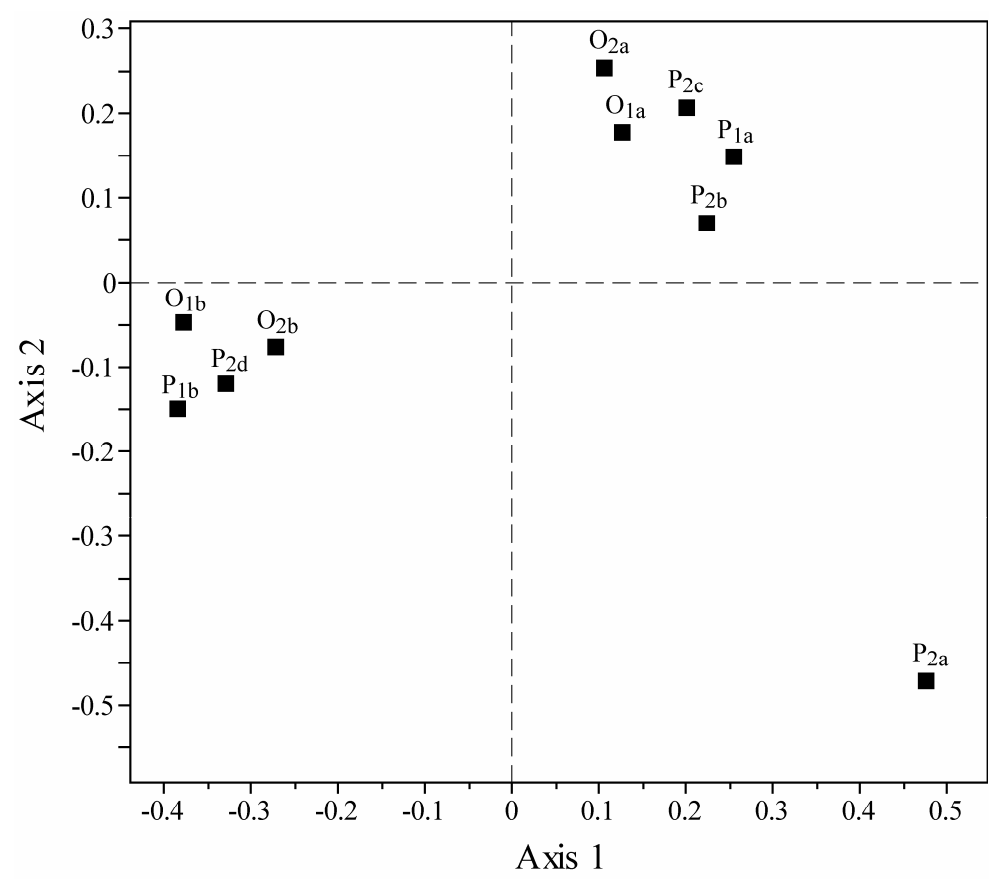

Figure 5. Ordination diagram of the considered plots for Ostia (sub-areas $O_{1}$ and $O_{2}$ ) and Marina di Palidoro (sub-areas $P_{1}$ and $P_{2}$ ). The variance associated to the first two axes of the principal coordinate analysis is $34.80 \%$ and $18.53 \%$, respectively. 
the inland for a length of $61 \mathrm{~m}$ (mean value) and PD is $21.5 \pm 16.1$ plants $\cdot \mathrm{m}^{-2}$ (mean of all the considered $\mathrm{P}$ plots). In particular, S. pungens, E. farctus and O. variegata have the highest PD $\left(6.0 \pm 2.4\right.$ plants $\mathrm{m}^{-2}$, mean value), followed by $C$. capitatus $\left(2.1 \pm 4.7\right.$ plants $\cdot \mathrm{m}^{-2}$, mean value), by $S$. colorata and A. maritima $(0.4 \pm 0.1$ plants $\cdot \mathrm{m}^{-2}$, mean value) and by E. spinosa, E. maritimum, M. marina, P. maritimum and C. soldanella (lower than 0.2 plants $\left.\cdot \mathrm{m}^{-2}\right)$. E. farctus and A. arenaria are rhizomatous species, with this last being the most important sand-fixing species with positive effects on sand stability $[18,34,42]$ and the mycorrhizal symbiosis playing an important role in the growth $[43,44]$.

The results underline the larger species presence along the gradient from the water-edge toward the inland in $\mathrm{P}$ compared to $\mathrm{O}$ where, on the contrary, plants exclusively colonize the inner areas due to the strong human disturbance which causes the fore-dune to become flat. Moreover, $\mathrm{P}_{2}$, characterised by a relatively lower disturbance than $\mathrm{P}_{1}$, has a $72 \%$ higher PD and a species distribution over a longer strip from water-edge toward the inland (53 and $73 \mathrm{~m}$ in $P_{1}$ and $P_{2}$, respectively). The study underlines the ability of the considered sand dune species to colonize the inner patch where microclimate and soil conditions are more favourable (by a $56 \%$ lower wind action and $12 \%$ higher SOM content, mean of the considered $\mathrm{O}$ and $\mathrm{P}$ plots) than the areas closer to the wateredge. In response to the more favourable conditions, $\mathrm{H}$ increases by $62 \%$ in the plots further from the wateredge.

\section{Conclusion}

An increase in human impact in the near future, along with global change, could act on sand dune species changing their tolerance to stress factors. A management plan needs to restore the most damaged dune areas and actively prevent further environmental impacts. This could be accomplished by developing and implementing strategies which reconcile demands for human recreation with conservation that is within the ecological carrying capacity of coastal dunes [45]. Vegetation is an important controlling factor for dune morphology [46]. An efficient conservative management would consist of a temporary protection of already degraded areas [2]. A combination of a large variety of driving forces might suggest an unsurmountable complexity. In fact every dune site has its own history and management policy which should incorporate this [22]. Moreover, the maintenance of coastal areas depends on the maintenance of native species [47]. Our results give information on the biodiversity of the Tyrrhenian coast including a natural protected area. The presence of the most important autoctonous sand dune species (on an average, 15, 15, 15 and 16 autoctonous species grow at $\mathrm{O}_{1}, \mathrm{O}_{2}, \mathrm{P}_{1}$ and $\mathrm{P}_{2}$, respectively) can provide information for restoring the perturbed dune areas when preparing management strategies. In particular, $A$. arenaria could have a positive effect on sand dune stabilization by contributing to plant colonization under favourable conditions [48]. It must be emphasized that species diversity and recovery capacity depend mostly on the undisturbed sand deposits in the foredunes by the limitation of human trampling and use of mechanical means and infrastructure development. This could favor sand deposits and consequently the species shift from the inland toward the water-edge, thus maintaining the coastal areas and their ecosystem services.

\section{REFERENCES}

[1] G. M. da Silva, P. Hesp, J. Peixoto and S. R. Dillenburg, "Foredune Vegetation Patterns and Alongshore Environmental Gradients: Mocambique Beach, Santa Catarina Island, Brazil," Earth Surface Processes and Landforms, Vol. 33, No. 10, 2008, pp. 1557-1573. http://dx.doi.org/10.1002/esp.1633

[2] A. Debez, K. B. Hamed, C. Grignon and C. Abdelly, "Salinity Effect on Germination, Growth, and Seed Production of the Halophyte Cakile maritime," Plant and Soil, Vol. 262, No. 1-2, 2004, pp. 179-189. http://dx.doi.org/10.1023/B:PLSO.0000037034.47247.67

[3] F. A. Donnelly and N. W. Pammenter, "Vegetation Zonation on a Natal Coastal Sand-Dune System in Relation to Salt Spray and Soil Salinity," South African Journal of Botany, Vol. 2, No. 1, 1983, pp. 46-51.

[4] M. Gilbert, N. Parameter and B. Ripley, "The Growth Responses of Coastal Dune Species Are Determined by Nutrient Limitation and Sand Burial," Oecologia, Vol. 156, No. 1, 2008, pp. 169-178. http://dx.doi.org/10.1007/s00442-008-0968-3

[5] A. M. Avis and R. A. Lubke, "The Effect of Wind Borne Sand and Salt Spray on the Growth of Scirpus nodosus in a Mobile Dune System," South African Journal of Botany, Vol. 1, No. 2, 1985, pp. 100-110.

[6] H. Yura and A. Ogura, "Sandblasting as a Possible Factor Controlling the Distribution of Plants on a Coastal Dune System," Plant Ecology, Vol. 185, No. 2, 2006, pp. 199208. http://dx.doi.org/10.1007/s11258-005-9095-y

[7] K. Pye and H. Tsoar, "Aeolian Sand and Sand Dunes," Springer, Berlin, 2009. http://dx.doi.org/10.1007/978-3-540-85910-9

[8] J. A. Stallins and A. J. Parker, "The Influence of Complex Systems Interactions on Barrier Island Dune Vegetation Pattern and Process," Annals of the Association of American Geographers, Vol. 93, No. 1, 2003, pp. 13-29. http://dx.doi.org/10.1111/1467-8306.93102

[9] L. Gratani, L. Varone and M. F. Crescente, "Photosynthetic Activity and Water Use Efficiency of Dune Species: The Influence of Temperature on Functioning," Photosynthetica, Vol. 47, No. 4, 2009, pp. 575-585. http://dx.doi.org/10.1007/s11099-009-0083-7 
[10] M. G. Barbour, "Life at the Leading Edge: The Beach Plant Syndrome," In: V. Seeliger, Ed., Coastal Plant Communities of America, Academic Press, New York, 1992, pp. 291-307.

[11] L. Gratani and G. Capannesi, "Cambiamenti in Vigore di Ammophila littoralis in Relazione Alla Sua Posizione Sulle Dune," Collana dei Rapporti Tecnici dell'ENEA, ENEA-RT/PAS/84/18, 1984, pp. 3-12.

[12] B. S. Ripley and N. W. Pammenter, "Do Low Standing Biomass and Leaf Area Index of Sub-Tropical Coastal Dunes Ensure that Plants Have an Adequate Supply of Water?" Oecologia, Vol. 139, No. 4, 2004, pp. 535-544. http://dx.doi.org/10.1007/s00442-004-1535-1

[13] R. Grunewald and H. Schubert, "The Definition of a New Plant Diversity Index ' $H$ 'dune' for Assessing Human Damage on Coastal Dunes-Derived from the Shannon index of Entropy $H^{\prime}$," Ecological Indicators, Vol. 7, No. 1, 2007, pp. 1-21. http://dx.doi.org/10.1016/j.ecolind.2005.09.003

[14] C. Hobohm, "Plant Species Diversity and Endemism on Islands and Archipelagos, with Special Reference to the Macaronesian Islands," Flora, Vol. 195, No. 1, 2000, pp. 9-24.

[15] D. Tilman, P. Reich, J. Knops, D. Wedin, T. Mielke and C. Lehman, "Diversity and Productivity in a Long-Term Grassland Experiment," Science, Vol. 294, No. 5543, 2001, pp. 843-845. http://dx.doi.org/10.1126/science.1060391

[16] N. M. Voronkova, E. V. Burkovskaya, T. A. Bezdeleva and O. L. Burundukova, "Morphological and Biological Features of Plants Related to Their Adaptation to Coastal Habitats," Russian Journal of Ecology, Vol. 39, No. 1, 2008, pp. 1-7. http://dx.doi.org/10.1134/S1067413608010013

[17] G. Baeyens and M. L. Martínez, "Animal Life on Coastal Dunes: From Exploitation and Prosecution to Protection and Monitoring," In: M. L. Martínez and N. P. Psuty, Eds., Coastal Dunes, Ecology and Conservation, Ecological Studies, Springer-Verlag, Berlin, 2004, pp. 279296. http://dx.doi.org/10.1007/978-3-540-74002-5_17

[18] S. Rodríguez-Echeverría and H. Freitas, "Diversity of AMF Associated to Ammophila arenaria ssp. arundinacea in Portuguese Sand Dunes," Mycorrhiza, Vol. 16, No. 8, 2006, pp. 543-552. http://dx.doi.org/10.1007/s00572-006-0070-9

[19] E. Van der Maarel, "Some Remarks on the Functions of European Coastal Ecosystems," Phytocoenologia, Vol. 33, No. 2-3, 2003, pp. 187-202. http://dx.doi.org/10.1127/0340-269X/2003/0033-0187

[20] E. Forey, B. Chapelet, Y. Vitasse, M. Tilquin, B. Touzard and R. Michalet, "The Relative Importance of Disturbance and Environmental Stress at Local and Regional Scales in French Coastal Sand Dunes," Journal of Vegetation Science, Vol. 19, No. 4, 2008, pp. 493-502. http://dx.doi.org/10.3170/2008-8-18392

[21] J. P. Grime, "Plant Strategies and Vegetation Processes," John Wiley and Sons, Chichester, 1979, 222p.

[22] S., Provoost, M. L. M. Jones and S. E. Edmondson,
"Changes in Landscape and Vegetation of Coastal Dunes in Northwest Europe: A Review," Journal of Coastal Conservation, Vol. 15, No. 1, 2011, pp. 207-226. http://dx.doi.org/10.1007/s11852-009-0068-5

[23] R. C. Kay and J. Alder, "Coastal Planning and Management," E\&F Spon, London, 2005.

[24] J. H. Connell, "Diversity in Tropical Rain Forests and Coral Reefs," Science, Vol. 199, No. 4335, 1978, pp. 1302-1310.

http://dx.doi.org/10.1126/science.199.4335.1302

[25] J. P. Grime, "Control of Species Diversity in Herbaceous Vegetation," Journal of Environmental Management, Vol. 1, No. 1, 1973, pp. 151-167.

[26] C. J. Lortie, R. W. Brooker, P. Choler, Z. Kikvidze, R. Michalet, F. I. Pugnaire and R. M. Callaway, "Rethinking plant community theory," Oikos, Vol. 107, No. 2, 2004, 433-438. http://dx.doi.org/10.1111/j.0030-1299.2004.13250.x

[27] R. H. MacArthur and E. O. Wilson, "The Theory of Island Biogeography," Princeton University Press, Princeton, 1967.

[28] C. Caputo, "Il Litorale Laziale da Capo Linaro ad Anzio: Caratteristiche Fisiografiche e Variazioni Recenti Della Linea di Riva," 19 Forum La Fascia Costiera ToscoLigure-Laziale, Forte dei Marmi, Italy, 1989, pp. 31-38.

[29] J. R. Sims and V. A. Haby, "Simplified Colorimetric Determination of Soil Organic Matter," Soil Science, Vol. 112, No. 2, 1971, pp. 137-141. http://dx.doi.org/10.1097/00010694-197108000-00007

[30] A. Walkley, "A Critical Examination of a Rapid Method for Determining Organic Carbon in Soils: Effects of Variations in Digestion Conditions and of Inorganic Soils Constituents," Soil Science, Vol. 63, No. 4, 1947, pp. 251-264. http://dx.doi.org/10.1097/00010694-194704000-00001

[31] L. Gratani, M. Amadori, C. Marinucci and M. Moriconi, "Study of the Relations between Biomass and Specific Cover in Psammophile Vegetation (Circeo National Park, Italy)," Annali di Botanica, Vol. 40, No. 1, 1982, pp. 97106.

[32] C. Marinucci and L. Gratani, "Cartografia Della Vegetazione e Della Biomassa," Annali di Botanica, Vol. 41, No. 1, 1983, pp. 113-131.

[33] J. S. Singh, W. K. Lauenroth and R. K. Sernhorst, "Review and Assessment of Various Techniques for Estimating Net Aerial Primary Production in Grasslands from Harvest Data," Botanical Review, Vol. 41, No. 2, 1975, pp. 181-232. http://dx.doi.org/10.1007/BF02860829

[34] E. Forey, C. J. Lortie and R. Michalet, "Spatial Patterns of Association at Local and Regional Scales in Coastal Sand Dune Communities," Journal of Vegetation Science, Vol. 20, No. 5, 2009, pp. 916-925. http://dx.doi.org/10.1111/j.1654-1103.2009.01095.x

[35] L. Gratani, M. Amadori and L. Veri, "A Preliminary Study of the Maquis at Castelporziano (Lazio) Structure and Biomass with the Use of the Phytosociological Table," Annali di Botanica, Vol. 40, No. 1, 1982, pp. 163-170. 
[36] P. Haase, F. L. Pugnaire, S. C. Clark and L. D. Incoll, "Spatial Patterns in a Two-Tiered Semi-Arid Shrubland in Southeastern Spain," Journal of Vegetation Science, Vol. 7, No. 4, 1996, pp. 527-534. http://dx.doi.org/10.2307/3236301

[37] S. Kefi, M. Rietkerk, C. L. Alados, Y. Pueyo, V. P. Papanastasis, A. ElAich and P. C. de Ruiter, "Spatial Vegetation Patterns and Imminent Desertification in Mediterranean Arid Ecosystems," Nature, Vol. 449, No. 7159, 2007, pp. 213-217. http://dx.doi.org/10.1038/nature06111

[38] D. Malkinson, R. Kadmon and D. Cohen, "Pattern Analysis in Successional Communities-An Approach for Studying Shifts in Ecological Interactions," Journal of Vegetation Science, Vol. 14, No. 2, 2003, pp. 213-222.

[39] R. Michalet, "Is Facilitation in Arid Environments the Result of Direct or Complex Interactions?" New Phytologist, Vol. 169, No. 1, 2006, pp. 3-6. http://dx.doi.org/10.1111/j.1468-8137.2006.01617.x

[40] M. G. Barbour and T. De Jong, "Response of West Coast Beach Taxa to Salt Spray, Seawater Inundation and Soil Salinity," Bulletin of the Torrey Botanical Club, Vol. 104, No. 1, 1977, pp. 29-34. http://dx.doi.org/10.2307/2484662

[41] D. Harris and A. J. Davy, "The Regenerative Potential of Elymus farctus from Rhizome Fragments and Seeds," Journal of Ecology, Vol. 74, No. 1, 1986, pp. 1057-1067. http://dx.doi.org/10.2307/2260233

[42] A. H. L. Huiskes, "Biological Flora of the British Isles: Ammophila arenaria (L.) Link," Journal of Ecology, Vol. 67, No. 1, 1979, pp. 363-382.

\section{http://dx.doi.org/10.2307/2259356}

[43] L. Gratani, "A Critical Approach to the Problem of the Vigour of Ammophila littoralis (Beauv.) Rothm.," Ecologia Mediterranea, Vol. 13, No. 1-2, 1987, pp. 53-60.

[44] J. V. Perumal and M. A. Maun, "The Role of Mycorrhizal Fungi in Growth Enhancement of Dune Plants Following Burial in Sand," Functional Ecology, Vol. 13, No. 4, 1999, pp. 560-566. http://dx.doi.org/10.1046/j.1365-2435.1999.00348.x

[45] L. M. C. Thompson and T. A. Schlacher, "Physical Damage to Coastal Dunes and Ecological Impacts Caused by Vehicle Tracks Associated with Beach Camping on Sandy Shores: A Case Study from Fraser Island, Australia," Journal of Coastal Conservation, Vol. 12, No. 2, 2008, pp. 67-82. http://dx.doi.org/10.1007/s11852-008-0032-9

[46] S. A. Wolfe and W. G. Nickling, "The Protective Role of Sparse Vegetation in Wind Erosion," Progress in Physical Geography, Vol. 17, No. 1, 1993, pp. 50-68. http://dx.doi.org/10.1177/030913339301700104

[47] M. W. Schwartz, C. A. Brigham, J. D. Hoeksema, K. G. Lyons, M. H. Mills and P. J. van Mantgem, "Linking Biodiversity to Ecosystem Function: Implications for Conservation Ecology," Oecologia, Vol. 122, No. 3, 2000, pp. 297-305. http://dx.doi.org/10.1007/s004420050035

[48] K. F. Nordstrom, N. L. Jackson, J. M. Hartman and M. Wong, "Aeolian Sediment Transport on a Human-Altered Foredune," Earth Surface Processes and Landforms, Vol. 32, No. 1, 2007, pp. 102-115. http://dx.doi.org/10.1002/esp.1377 